\title{
Laboreal
}

Volume $12 \mathrm{~N}^{\circ} 2$ | 2016

Equipamentos de Proteção Individual II

\section{A contaminação por agrotóxicos e o uso de EPI: análise de aspectos legais e de projeto}

La contaminación por pesticidas y el uso de EPP: analisis de los aspectos legales y proyecto

La contamination par les pesticides et l'utilisation des EPI: analyse des aspects de la législation et de la conception

Pesticide Contamination and use of PPE: analyzing legal aspects and design

Luiz Antonio Meirelles, Marcelo Motta Veiga e Francisco Duarte

\section{(e) OpenEdition}

Journals

Edição electrónica

URL: http://journals.openedition.org/laboreal/2472

DOI: $10.4000 /$ laboreal.2472

ISSN: 1646-5237

\section{Editora}

Universidade do Porto

\section{Refêrencia eletrónica}

Luiz Antonio Meirelles, Marcelo Motta Veiga e Francisco Duarte, «A contaminação por agrotóxicos e o uso de EPI: análise de aspectos legais e de projeto », Laboreal [Online], Volume 12 №2 | 2016, posto online no dia 01 dezembro 2016, consultado o 08 outubro 2019. URL : http://journals.openedition.org/ laboreal/2472 ; DOI : 10.4000/laboreal.2472

Este documento foi criado de forma automática no dia 8 outubro 2019 


\section{A contaminação por agrotóxicos e o uso de EPI: análise de aspectos legais e de projeto}

La contaminación por pesticidas y el uso de EPP: analisis de los aspectos legales y proyecto

La contamination par les pesticides et l'utilisation des EPI: analyse des aspects de la législation et de la conception

Pesticide Contamination and use of PPE: analyzing legal aspects and design

Luiz Antonio Meirelles, Marcelo Motta Veiga e Francisco Duarte

\section{NOTA DO EDITOR}

http://dx.doi.org/10.15667/laborealxii0216lam

Manuscrito recebido em: março/2016

Aceite após peritagem: maio/2016

\section{I - Introdução}

1 Atividades laborais perigosas e insalubres são parte do dia-a-dia de muitos trabalhadores. As medidas de prevenção e de proteção contra acidentes e doenças nos locais de trabalho visam eliminar ou reduzir os riscos para a saúde e melhorar a segurança dos trabalhadores na sua origem, na geração do risco e nas suas eventuais consequências. A prevenção deve ser sempre priorizada em relação às medidas de proteção e de mitigação das consequências. Nas situações de processos de trabalho em que as medidas de prevenção implantadas não foram suficientes e que medidas adicionais sejam muito onerosas, medidas de proteção podem ser mais econômicas e viáveis. 
2 Assim, o uso de equipamentos de proteção coletiva (EPC) no ambiente de trabalho, ou de EPI nos corpos dos trabalhadores, atenderia a essa busca pela viabilidade financeira, assumindo-se que eventuais mudanças nos projetos de equipamentos, nas instalações ou nos materiais que causam riscos sejam mais onerosas para o empregador. Assume-se que depois que as situações causadoras dos riscos já estão efetivamente incorporadas, intervenções que exijam prevenção primária (eliminação do risco) são mais dispendiosas do que intervenções que demandem prevenção secundária (redução da probabilidade), protetivas (sobre os efeitos) e mitigatórias (sobre as consequencias) de eventuais danos aos trabalhadores.

3 Nesse sentido, o uso de equipamento de proteção individual (EPI) faz parte da rotina diária de muitos trabalhadores. Desde a sua concepção, muitos projetos de processos de trabalho já incorporam o uso de EPI como medida protetiva, buscando reduzir ou atenuar os riscos à saúde e à segurança dos trabalhadores. Os EPI não são projetados para eliminar os riscos. Por isso, considera-se como boa prática de projeto de EPI, que estes sejam valorizados apenas como soluções provisórias, devendo-se evitar o uso de EPI como solução permanente.

4 No tocante ao projeto, é notável a predominância nos EPI comercializados e na literatura, de projetos orientados para agentes isolados. Essa orientação dos projetos de EPI para um ou poucos agentes isolados, sem levar em conta o conjunto de agentes a que o trabalhador estaria exposto nas suas atividades, pode ser interpretada como uma falha de concepção dos EPI, i.e., a exposição real do trabalhador aos múltiplos agentes nas diferentes situações de trabalho, não é considerada no projeto dos EPI.

5 No entanto, o estado da técnica [1] já contempla soluções para EPI por agentes simultâneos. Nesse sentido podem ser citados: (i) pedido de patente no Brasil que descreve um "Respirador com peça facial filtrante tendo recorte para o nariz"; (ii) a norma técnica brasileira NBR 8221 que dispõe sobre a proteção contra choques mecânicos por "Capacetes de segurança para uso na indústria" e (iii) na patente americana (US 9.211.069) que trata de EPI com monitoramento fisiológico integrado.

6 Em muitos casos, como o do mergulhador comercial, os EPI deveriam proteger o trabalhador, simultaneamente, da exposição aos vários agentes agressivos, vedando, protegendo contra hipotermia, abastecendo o trabalhador de ar, e facilitando o uso de ferramentas para corte, solda, montagem e desmontagem, etc. Logo, constata-se uma lacuna entre o Estado da Técnica e as práticas predominantes nos projetos de EPI.

7 A literatura acadêmica já mostrou várias falhas na concepção e nos projetos dos EPI, sugerindo a necessidade de revisões tecnológicas de amplo alcance. Alguns exemplos de estudos que retratam esses problemas são:

a. Inadequação antropométrica - Dados antropométricos carecem de acompanhamento regular. Na Noruega (Bolstad, Benum \& Rokne, 2001) havia falta de dados antropométricos necessários para projetos de EPI ou os mesmos não estavam atualizados. Nos Estados Unidos da América (Onyebeke et al, 2016), os EPI utilizados na indústria da Construção Civil, como luvas e botas, são inadequados antropometricamente para mulheres.

b. Carga Fisiológica - Experimentos com homens e mulheres usando EPI empregados por bombeiros (Taylor, Lewis, Notley \& Peoples, 2012) resultaram em medidas fisiológicas discrepantes estatisticamente entre a situação de controle e a de uso dos EPI, em atividades de andar e subir degraus, com uso de botas, roupas de proteção e respiradores autônomos. As atividades realizadas com EPI tiveram maior carga fisiológica. 
c. Especificações de projeto de EPI insuficientes - Estudos com pilotos da aviação agrícola (Fonseca, Zeigelboim, Lacerda, Ribas \& Spanhol, 2016) mostraram que estes usavam simultaneamente tampões para ouvidos juntamente com capacetes também previstos para proteção auricular. Ainda assim apresentaram perdas de acuidade auditiva associáveis a exposição ao ruído no trabalho.

d. Necessidade de revalidação em campo de testes laboratoriais - 0 pressuposto de que o uso do EPI neutraliza o risco está em questão para o caso do ruído. Em um estudo de desempenho de protetores auriculares em campo, com microfones inseridos nos EPI (Nélisse, Gaudreau, Boutin, Voix \& Laville, 2012), constatou-se problemas na atenuação dos ruídos e de montagem dos tampões de ouvido. Além disso, a eficiência medida em campo foi inferior a do laboratório. Um estudo de audiometria de trabalhadores nos EUA concluiu que protetores auriculares foram inefiientes em evitar a perda de acuidade auditiva; os resultados encontrados enfatizaram a importância de eliminar a exposição perigosa de ruídos, em vez de depender do uso de protetores auditivos (Groenewold, Masterson, Themann \& Davis, 2014).

e. Conforto térmico - Na agricultura brasileira, os problemas de inadequação dos EPI estão presentes, sendo freqüente encontrar trabalhadores rurais não usando EPI obrigatórios durante o manuseio e aplicação de produtos químicos agrícolas. Uma das razões apontadas é que muitos dos EPI causam desconforto térmico, que em casos extremos pode mesmo levar ao estresse de calor (Veiga, Duarte, Meirelles, Garrigou \& Baldi, 2007; Almeida, Veiga, Duarte, Meirelles \& Veiga, 2012). Crockford (1999) mostrou que o uso de EPI em regiões tropicais, especialmente na agricultura a céu aberto, agrava problemas de desconforto térmico. $O$ uso do equipamento influencia na termo-regulação, podendo dificultar a perda ou a acumulação de calor pelo corpo humano. Por isso, em atividades em que a utilização de EPI seja essencial, existe uma probabilidade maior de problemas para a saúde provocadas pelo calor.

f. Permeabilidade - O EPI é permeável ao agrotóxico. As observações no uso de EPI demonstraram que não é eficiente para evitar a contaminação. Além disso, alguns fatores contribuem para aumento da permeabilidade dos EPI, como o número de lavagens, o detergente utilizado nas lavagens e a presença e tipo de costuras das vestimentas (Veiga \& Melo, 2016; Garrigou, Baldi, Le Frious, Anselm \& Vallier, 2011). Estudos sobre luvas usadas em pintura por spray (Ceballos, Reeb-Whitaker, Sasakura, Dills \& Yost, 2015) mostraram que permeabilidade de luvas de látex ou de borracha nitrílica de $0,13 \mathrm{~mm}$ de espessura não fornecem proteção contra 10 solventes testados.

8 Os fabricantes de EPI agrícolas, devido a sua baixa participação no mercado total dos EPI, simplesmente adaptam os EPI utilizados na indústria, procurando atender as normas de certificação e aos testes laboratoriais. Na fase de projeto do EPI, a escolha de qual agente isolado proteger está associada ao cumprimento da legislação que prescreve e certifica os EPI fabricados através de testes em laboratórios credenciados. Portanto, a legislação é a diretriz principal para os projetos de novos EPI, sendo fator fundamental para que os fabricantes definam características, especificações e qual(ais) a(s) função(ões) principal(is) de cada EPI.

\section{II - O EPI e a legislação brasileira}

9 No Brasil, a legislação do Ministério do Trabalho relativa à Higiene, Segurança e Medicina do Trabalho torna obrigatória um conjunto de normas regulamentadoras que condicionam os empregadores a selecionar, adquirir e distribuir para os trabalhadores 
os EPI como alternativa ao pagamento de adicionais de insalubridade (Meirelles, Veiga \& Duarte, 2012).

10 A Norma Regulamentadora 15 (NR-15) define as atividades ou operações insalubres como as que expõem os trabalhadores a agentes passíveis de gerar "danos à saúde do trabalhador, durante sua vida laboral". Se a insalubridade não é eliminada ou neutralizada, os empregadores devem pagar um adicional ao salário dos empregados expostos. 0 espírito da lei é estimular financeiramente os empregadores a eliminarem e controlarem os riscos no ambiente de trabalho de modo a preservar a saúde dos trabalhadores.

11 Desta forma, a exposição do trabalhador a fatores insalúbres implica em um montante a ser pago mensalmente a cada trabalhador, a título de adicional de insalubridade, na proporção de um percentual sobre o salário mínimo regional.

12 Ingenuamente, a legislação assume que o simples uso de EPI elimina ou neutraliza a insalubridade, sendo elemento para dispensar o empregador do pagamento do adicional de insalubridade (Meirelles et al, 2012).

13 Segundo prescrição legal, os EPI são diferenciados de acordo com a região do corpo, órgãos ou sentidos que visam proteger (visão, audição, sistema respiratório, pele, ...). 0 Ministério do Trabalho é a autoridade pública competente para emitir os Certificados de Aprovação dos projetos dos EPI, credenciando laboratórios e autorizando sua produção e venda.

14 A legislação identifica agentes agressivos isolados como fatores de insalubridade, sem nexos com atividades específicas. A única referência a atividades está limitada a associações a graus de insalubridade, mas não aos EPI que em tese protegeriam o trabalhador. A obrigação de relacionar os EPI com as atividades é delegada ao empregador, que efetivamente compra os EPI.

Por responsabilidade direta, se o empregador não fornecer ou não fiscalizar o uso dos EPI, deve, por força de lei, pagar ao trabalhador um adicional de insalubridade. Se o EPI é fornecido e seu uso é fiscalizado, o empregador está legalmente dispensado do pagamento do adicional de insalubridade. Portanto, há uma clara associação entre o valor do adicional de insalubridade e os custos de EPI para uso em locais de trabalho.

16 A legislação não exige pagamento cumulativo de adicionais de insalubridade, relativo a exposição a diferentes agentes, simultaneamente. Paga-se o adicional de insalubridade apenas para o agente que represente o maior valor. Assim, o EPI aprovado para proteger o trabalhador em atividades insalúbres está relacionado a cada agente separadamente.

17 A lógica financeira sugere ao empregador fornecer EPI aos trabalhadores somente quando o custo dos EPI for menor que o valor pago a título de adicional de insalubridade; caso contrário seria mais vantajoso para o empregador pagar o adicional de insalubridade (Meirelles et al, 2012).

o adicional de insalubridade não contempla efeitos sinérgicos, o que pode ser considerado uma deficiência legal. Caso houvesse essa prescrição legal, seria necessário desenvolver equipamentos protetivos (conjunto de EPI) que fossem funcionalmente mais eficientes e pudessem ser utilizados para proteger contra diversos agentes simultaneamente. A adoção progressiva deste tipo de EPI resultaria numa proteção mais efetiva. Contudo, se o custo do conjunto destes EPI ultrapassar o valor do adicional 
de insalubridade correspondente ao agente de maior valor de adicional, pode haver uma resistência para sua comercialização e implantação (Meirelles et al, 2012).

Portanto, a discrepância entre o estado da técnica e das práticas vigentes é influenciada pela legislação em vigor. Uma prática de gestão freqüente é o atendimento dos requisitos mínimos da normas legais, privilegiando soluções de menor custo financeiro, desde que atendam aos padrões legais estabelecidos.

Contudo, a formulação de normas legais não inclui necessariamente uma atualização de conhecimentos científicos e tecnológicos. No entanto, gradativamente, a legislação poderia incorporar novos conhecimentos científicos e tecnológicos.

\section{III - O uso de EPI na agricultura}

21 A contaminação por agrotóxicos expõe diretamente os trabalhadores rurais ao longo de toda sua jornada de trabalho. Estes trabalhadores estão mais expostos a contaminações por problemas de projeto nos EPI.

22 A análise crítica de situações reais mostrou problemas de concepção dos EPI agravados pelo uso continuado, levando a contaminação dos trabalhadores em atividades de preparação, aplicação de agrotóxicos, colocação e remoção dos EPI do corpo, limpeza dos EPI e armazenamento dos mesmos após o uso.

Em um estudo de caso sobre o processo de trabalho rural na cultura de tomate no Brasil, observou-se que o uso de EPI foi a única solução adotada para a proteção à saúde e à segurança dos trabalhadores (Veiga et al., 2007).

24 A queixa mais freqüente dos trabalhadores rurais em relação ao uso dos EPI é o desconforto térmico associado às roupas de proteção, sobretudo em dias quentes. Os EPI reduzem a circulação de ar no corpo do trabalhador, ocasionando um verdadeiro "efeito estufa". Um trabalhador rural brasileiro chega a trabalhar longas horas, em temperaturas superiores a $40^{\circ} \mathrm{C}$, com humidade superior a $90 \%$, numa condição de trabalho bastante insalubre, que pode trazer sérias conseqüências negativas à sua saúde.

Garrigou et al (2011) mostraram que os EPI não protegiam os trabalhadores rurais de forma eficiente e poderiam até mesmo ser a uma fonte de contaminação. Os trabalhadores rurais estudados se contaminavam enquanto se vestiam, despiam ou lavavam seus EPI. Além disso, percebeu-se a ineficiência da permeabilidade do material de vedação dos EPI. Os agrotóxicos poderiam facilmente penetrar através do revestimento dos EPI em questão de minutos, especialmente após muitas lavagens.

Nesses casos, observou-se que os trabalhadores rurais não seguiam, por diversos motivos, as recomendações quanto a manutenção, lavagem, vestir/despir, descarte e armazenagem dos EPI. Essas situações se constituíam em fontes primárias de contaminação. Além disso, essa contaminação dos trabalhadores poderia se propagar, pois muitos desses trabalhadores levavam para sua residência as suas roupas e os EPI que usavam para lavar. Os EPI são lavados, secos e guardados ao alcance das pessoas com quem eventualmente compartilhavam suas residências, inclusive crianças.

Por isso, foi constatado que em diversas situações o uso dos EPI aumentou a contaminação por agrotóxicos dos trabalhadores. Pressupôs-se que as deficiências de projeto e produção resultaram em EPI inadequados e desconfortáveis. Assim, as objeções dos trabalhadores rurais à utilização desses equipamentos encontram 
evidências empíricas e fundamentos concretos (Baldi, Lebailly, Rougetet, Dulaurent \& Marquer, 2006).

28 As orientações sobre métodos de uso, manutenção e armazenagem dos EPI não são validadas em campo, antes da emissão dos Certificados de Aprovação, nos diversos tipos de situações de trabalho. Em conseqüência, os métodos prescritos não correspondem aos métodos praticados. $O$ descarte dos EPI também não parece ser contemplado nos projetos. Os materiais empregados não são biodegradáveis nem biodestrutíveis. 0 descarte usual desses equipamentos como lixo comum sugere a ausência de avaliação do perigo representado pela concentração de materiais tóxicos neles presentes. Carecem de avaliação os efeitos decorrentes da adoção da queima de EPI em fogueiras ou do enterro, constatadas no estudo de caso apresentado em Veiga et al. (2007).

\section{IV - Discussão}

Situações de trabalho penosas e insalubres são realidades vivenciadas por muitos trabalhadores. A prevenção [2] deveria ser priorizada em relação a medidas de proteção ou atenuação dos riscos ou dos perigos. Na impossibilidade de prevenção, a legislação deveria estimular medidas de proteção coletiva. $\mathrm{Na}$ falta de um estímulo legal para a adoção de medidas de prevenção ou de proteção coletiva como solução financeiramente viável para controlar situações insalubres ou perigosas, o que ocorre na quase totalidade dos casos é a utilização dos EPI como solução "paliativa" temporária (Meirelles et al, 2012).

Especialmente nas situações laborais agrícolas, medidas protetivas coletivas são pouco utilizadas, devido ao seu custo elevado e difícil adaptabilidade às condições de campo. Na maioria das situações, "proteger" é mais "econômico" para o empregador do que prevenir. Logo, no dia-a-dia das organizações, o uso de EPI se torna parte da rotina permanente de muitos trabalhadores.

31 Ao incorporar essa falha como inevitável, a maioria dos projetos de Saúde e Segurança no Trabalho (SST) já reconhece que não é possível evitar todos os riscos, e, por isso, contempla, desde a sua fase de concepção, a utilização de EPI. Este fato é um reconhecimento da existência de falhas do tipo organizacional ou latentes em relação ao processo de trabalho e de mecanismos de prevenção. Estas falhas seriam introduzidas nos sistemas por níveis hierárquicos superiores e estão relacionadas à gestão ou concepção dos sistemas produtivos (Reason, 2004).

No caso dos EPI, essas falhas estão relacionadas ao projeto, ao arcabouço jurídico, à certificação e à colocação desses equipamentos de proteção no mercado. Ressalta-se que, os EPI têm apenas a finalidade de proteger, não de evitar, os riscos à saúde e à segurança dos trabalhadores. Por isso, a Organização Mundial do Trabalho alerta que a utilização de EPI só deve ser considerada como um dos últimos recursos de tecnologia de proteção/controle de riscos aos trabalhadores (ILO, 2001).

Os casos das falhas apresentadas do uso de EPI na agricultura colocam em questão a ineficácia dos EPI quando analisados em situações reais. A maioria dos EPI foi concebida para situações de utilização que não correspondem às condições reais de trabalho. Essas falhas do tipo organizacional têm implicação sobre a ação da maior parte das atores competentes em termos de prevenção. Coloca-se em evidência a necessidade de novas 
normalizações sobre EPI para a agricultura, o que já começa a ocorrer em algumas localidades, e.g. na comunidade européia (Meirelles et al, 2012).

Nesse sentido, falhas envolvendo a concepção e o uso de EPI podem ser consideradas como parte de um problema maior, sistêmico e clássico de transferência de tecnologias (Wisner, 1997; Palis, 2006).

Ressalta-se que a principal função do EPI permanece como de reduzir o risco ou mitigar a conseqüência, o que diverge de uma expectativa legal favorável à saúde e à segurança do trabalhador. Essa valorização da proteção em detrimento da prevenção fica evidenciada quando se percebe que existe um número cada vez maior de projetos de processos de trabalho incompletos que já incorporam na sua concepção a utilização de EPI para encobrir suas falhas latentes. Melhores legislações poderiam ser uma tentativa de corrigir parte dessas falhas organizacionais e de concepção.

Um indicador dessa falta de incentivos para medidas de prevenção é a carência de estudos sobre desenvolvimento tecnológico de EPI. $O$ estado da técnica dos EPI está bem distante da utilização desses equipamentos em situações reais. Neste contexto, grande parte dos EPI não seria adequada à sua utilização e/ou finalidade. Na maioria das vezes, parece que os EPI agrícolas além de não conseguir evitar a contaminação, o acidente ou a lesão, podem ser, inclusive, como no caso dos estudos aqui discutidos, fonte primária de contaminação dos trabalhadores e de seus familiares (Meirelles et al, 2012).

Além das dificuldades decorrentes do emprego de metodologias de projeto de produto obsoletas, as indicações para uso e manutenção dos EPI atendem a sugestões de Serviços de Saúde e Segurança no Trabalho (SST) e a indicações em rótulos e manuais de instruções elaborados por fabricantes diversos, sem validação das indicações em testes de campo e sem referências para avaliação da adequação do estado do produto. EPI são concebidos como produtos descartáveis, mas não são encontradas usualmente indicações de métodos de avaliação do fim da sua vida útil (Medeiros, 1995).

Portanto, as lacunas funcionais no projeto, na concepção, no uso, na manutenção, no armazenamento e no descarte dos EPI empregados nos estudos de caso analisados comprometeram a saúde humana, a qualidade da vida no trabalho rural e agravaram os problemas ambientais. A prevenção dos riscos e perigos à saúde humana e ao meio ambiente é um benefício e, como tal, tem custos. Se não resolvida no projeto do processo de trabalho, restam a proteção coletiva e, por último, a proteção individual. Mas é preciso que essa proteção seja eficaz, o que não aconteceu nos casos aqui estudados.

A presunção de que o EPI deve eliminar ou neutralizar a insalubridade para quaisquer situações concretas, associada à necessidade de se produzirem EPI de baixos custos, e ainda manter a vantagem financeira para os empregadores de proteger em detrimento de prevenir, cria alguns condicionantes legais que parecem imbricados a elementos técnicos relevantes para os projetos de EPI:

1. Exclusão de efeitos sinérgicos - a legislação brasileira não prevê acumulação de pagamentos de adicionais de insalubridade pela exposição simultânea a diferentes "fatores de insalubridade". Um trabalhador exposto a vários agentes recebe adicional somente de um agente - o de grau mais elevado. A intenção legal deveria estimular financeiramente a adoção de padrões mais eficientes de higiene e segurança. Contudo, qualquer iniciativa é prejudicada pela possibilidade legal do empregador pagar apenas por um fator de insalubridade, sem considerar o conjunto dos efeitos sinérgicos danosos da exposição a diversos agentes agressivos. 
2. Certificação dos EPI exclusivamente por testes laboratoriais - o Ministério do Trabalho emite Certificados de Aprovação. Os processos de validação dos projetos de EPI condicionam os fabricantes a desenvolver em EPI adequados aos ensaios a que serão submetidos nos laboratórios especializados. Não há avaliação da efetividade funcional dos EPI. Assim, verifica-se a ocorrência de condições simultâneas para a limitação de preços e para o desenvolvimento de projetos. $O$ resultado deste conflito de interesses é que os projetos dos EPI são orientados para aprovação em ensaios laboratoriais e não para o uso.

3. Neutralização de risco - conforme pressuposto legal, o uso de EPI neutralizaria os riscos. Não há exigência legal de que um laudo técnico comprove a neutralização dos riscos pelos EPI seja emitido com base em experimentos nas situações reais. Sem experimentos em campo, a neutralização dos riscos será sempre presumida. Nos casos concretos, os EPI são avaliados com base nas especificações nominais constates dos Certificados de Aprovação, e.g. para um nível de ruído real em um local de trabalho medido de $120 \mathrm{db}$, assume-se que com o uso de um EPI que atenue mais que $35 \mathrm{db}$ o trabalhador estaria protegido, pois manteria a exposição nos limites de tolerância legal de $85 \mathrm{db}$.

4. Termo de Responsabilidade - No caso de falta de laboratórios especializados credenciados pelo Ministério do Trabalho para testes específicos de determinadas características dos EPI, a legislação dispõe que o EPI pode ser produzido e comercializado apenas com um Termo de Responsabilidade Técnica assinado pelo fabricante. A qualidade desses EPI aprovados com base em termos de responsabilidade não é avaliada em laboratórios e nem em situações reais de uso.

Por isso, faz-se necessário que as legislações sobre Saúde e Segurança no Trabalho (SST) disponham de mecanismos que possam incentivar medidas de prevenção e proteção coletiva por parte dos empregadores, evitando estimular a produção de EPI de baixo custo e direcionados apenas para proteger contra agentes isolados.

\section{V - Considerações finais}

41 Este estudo demonstrou que o risco de contaminação por agrotóxicos dos trabalhadores rurais depende da eficiência do EPI. O estado das práticas de projetos de EPI para atividades agrícolas não considera o uso desses produtos em situações reais. Muito embora, o estado da técnica já contemple o efeito sinérgico dos diferentes contaminantes, tal não é ainda levado em conta nos projetos dos EPI comercializados no Brasil.

Muitos itens dos vestuários de proteção para a atividade agrícola são inadequados para o uso pretendido e não cumprem a função de evitar a contaminação. Por vezes, podem ser a própria fonte de contaminação. Logo, ratifica-se a função principal do EPI de apenas reduzir o risco ou mitigar as consequências, o que diverge de uma expectativa legal mais benéfíca para à saúde e segurança do trabalhador.

Nesse estudo foram analisados diversos problemas de concepção dos EPI agrícolas. Uma causa importante dessas falhas se deve ao fato de que os EPI comercializados foram projetados para proteger contra agentes agressivos isolados. Os EPI não consideram a atividade de trabalho em condições reais, que integra diferentes formas de contaminação, restrições e fatores de risco que agem sinergicamente.

Nesse sentido, o EPI tem por função principal proteger o usuário de eventuais danos à saúde devidos a exposição a agentes isolados, não sendo concebidos para uso simultâneo, tais como: luvas que não se integram com a vedação das mangas das 
roupas; e protetores auriculares do tipo concha que não prevêem o uso simultâneo das hastes de óculos de segurança.

Além disso, esses problemas são agravados porque os EPI empregados na agricultura foram adaptados da indústria sem revisão das especificações, e.g. EPI para proteção contra produtos químicos utilizados em uma cabine de pintura climatizada pode ser o mesmo utilizado na aplicação de agrotóxicos numa cultura de tomate.

Essas falhas de concepção e de projeto são colocadas em teste quando os EPI aprovados para indústria e com Certificados de Aprovação emitidos pelo Ministério do Trabalho são então comercializados para uso obrigatório na agricultura, sob pena de punição dos empregadores.

A expectativa é que os trabalhadores rurais adoeçam graças à precariedade dos projetos de EPI inadequados para o conjunto de agentes agressivos presentes nas suas situações reais de trabalho.

Cabe à legislação fornecer mecanismos que incentivem a prevenção e estimulem medidas de proteção coletiva por parte dos empregadores. No entanto, especialmente em situações agrícolas reais, estas medidas são muitas vezes simplesmente não utilizadas, por uma série de condicionantes, entre os quais o custo de projetos de sistemas de prevenção específicos para condições do trabalho agrícola.

Há pelo menos dois indicadores da falta de incentivos a medidas preventivas - a escassez de estudos sobre o desenvolvimento tecnológico dos EPI e o pressuposto, na legislação brasileira, que o uso de EPI seria suficiente para evitar que condições insalubres afetassem a saúde do trabalhador.

Esta falta de estímulo para prevenir e controlar condições insalubres e perigosas incentiva o uso de EPI como uma solução duradoura; não paliativa e temporária, como determinação legal. A utilização de EPI ineficientes vai perdurar, enquanto essa for a solução financeiramente mais vantajosa prevista em lei.

51 Portanto, a legislação brasileira deve ser aprimorada de forma a contemplar conjuntos de atividades específicas ao invés de apenas relacionar "fatores de insalubridade" e passar a exigir, como condição para emissão de Certificados de Aprovação dos EPI, a análise da atividade em condições reais e requerer testes de campo a partir de uma abordagem sóciotécnica e ambiental, com a participação de representantes de usuários, trabalhadores, instituições e autoridades públicas e fabricantes.

\section{BIBLIOGRAFIA}

Almeida, R.A.C.S., Veiga, M.M., Duarte, F.J.C.M., Meirelles, L.A., \& Veiga, L.B.E. (2012). Thermal comfort and personal protective equipment (PPE). Work (Reading, MA), 41, 4979-4982.

Baldi, I., Lebailly, P., Jean, S., Rougetet, L., Dulaurent, S., \& Marquet, P. (2006). Pesticide contamination of workers in vineyards in France. Journal of Exposure Analysis and Environmental Epidemiology, 16 (2), 115-124. 
Bolstad, G., Benum, B. \& Rokne, A., (2001). Anthropometry of Norwegian light industry and office workers. Applied Ergonomics, 32, 239-246.

Ceballos, D., Reeb-Whitaker, C., Sasakura, M., Dills, R., \& Yost, M. (2015). Protection efficacy of gloves against components of the solvent in a sprayed isocyanate coating utilizing a reciprocating permeation panel. The Annals of Occupational. Hygiene, 59(3), 358-372. doi:10.1093/ annhyg/meu099.

Crockford, C.W. (1999). Protective clothing and heat stress: introduction. The Annals of Occupational. Hygiene, 43(5), 287-288.

Fonseca, V.R., Zeigelboim, B.S., Lacerda, A.B.M., Ribas, A., \& Spanhol, G. (2016). Hearing Health in Agricultural Aviation Pilots from Cindacta II Wearing Earplugs and a Helmet. International Archives of Otorhinolaryngology, 20(2), 105-108.

Garrigou, A., Baldi, I., Le Frious, P., Anselm, R., \& Vallier, M. (2011). Ergonomics contribution to chemical risks prevention: An ergotoxicological investigation of the effectiveness of coverall against plant pest risk in viticulture. Applied Ergonomics, 42(2), 321-330. doi: 10.1016/j.apergo. 2010.08.001.

Groenewold, M.R., Masterson, E.A., Themann, C.L., \& Davis, R.R. (2014). Do Hearing Protectors Protect Hearing? American Journal of Industrial Medicine, 57, 1001-1010.

International Labour Office (ILO). (2001). Guidelines on occupational safety and health management systems, Geneva: ILO-OSH.

Medeiros, E.N. (1995) Análise de aspecto do gerenciamento do design de produtos em processos de modernização tecnológica sob o enfoque ergonômico. Tese de Doutorado - Coordenação dos Programas de Pós-graduação de Engenharia, Universidade Federal do Rio de Janeiro, Rio de Janeiro.

Meirelles, L.A., Veiga, M.M., \& Duarte, F.J.C.M. (2012). Efficiency of personal protective equipment used in agriculture. Work (Reading, MA), 41, 14-18.

Nélisse, H., Gaudreau, M.A., Boutin, J., Voix, J., \& Laville, F. (2012). Measurement of hearing protection devices performance in the workplace during full-shift working operations. The Annals of Occupational. Hygiene, Vol. 56(2), 221-232. doi:10.1093/annhyg/mer087.

Onyebeke, L.C., Papazaharias, D.M., Freund, A., Dropkin, J., McCann, M., Sanchez, S. H.,... Zuckerman, N.C. (2016). Access to Properly Fitting Personal Protective Equipment for Female Construction Workers. American Journal of Industrial Medecine. 59(11), 1032-1040. doi: 10.1002/ajim. 22624.

Palis, F.G. (2006). Our farmers at risks: behavior and belief system in pesticide safety. Journal of Public Health, 28(1), 43-48. doi:10.1093/pubmed/fdi06.

Reason, J. (2004) Managing the risks of organizational accidents. Aldershot: Ashgate.

Taylor, N.A.S., Lewis, M.C., Notley, S.R., \& Peoples, G.E. (2012). A fractionation of the physiological burden of the personal protective equipment worn by firefighters. European Journal of Applied Physiology. (112), 2913-2921. Doi: 10.1007/s00421-011-2267-7.

Veiga, M.M., Duarte, F.J.C.M., Meirelles, L.A., Garrigou, A., \& Baldi, I. (2007). A contaminação por agrotóxicos e os equipamentos de proteção individual (EPIs). Revista Brasileira de Saúde Ocupacional, 32(116), 57-68.

Veiga, M.M., \& Melo, C. (2016). Análise da eficiência dos equipamentos de proteção aos agrotóxicos utilizados em saúde pública. Laboreal, 12(1), 53-62. doi: 10.15667/

LABOREALXIIO116MMV 
Wisner, A. (1997). Ergotoxicologie dans les pays tropicaux. In: A. Wisner et al. Anthropotechnologie - vers un monde industriel pluricentrique (pp. 179-180). Toulouse: Octares Editions.

\section{NOTAS}

1. O estado da técnica é definido no Código de Propriedade Industrial do Brasil como "constituído por tudo aquilo tornado acessível ao público antes da data de depósito do pedido de patente, por descrição escrita ou oral, por uso ou qualquer outro meio, no Brasil ou no exterior..." (Brasil, Lei $\mathrm{n}^{\circ}$ 9.2 As medidas preventivas seriam aquelas que eliminam ou reduzem os riscos e perigos, atuando na sua fonte, ou seja, evitam e/ou reduzem a geração do risco ou do perigo.79, de 14 de maio de 1996, artigo 11).

2.

\section{RESUMOS}

Esse artigo analisa o uso dos Equipamentos de Proteção Individuais (EPI) na agricultura no Brasil. A (in)adequação desses equipamentos é evidente. Além de não protegerem integralmente o trabalhador contra o agrotóxico, ainda agravam os riscos. Os EPI não eliminam a insalubridade conforme determina a legislação e ainda aumentam a probabilidade de contaminação dos trabalhadores rurais em algumas atividades. Discutem-se aspectos da validação legal dos EPI. Esse processo de validação legal para fabricação e venda de EPI consiste em testes em laboratórios, por agentes agressivos isolados, o que pode ser considerado uma lacuna no processo de concepção. A validação legal não exige a análise das atividades típicas e/ou experimentos em situações de utilização mais frequentes ou mais críticas para a saúde do trabalhador.

En este artículo se analiza el uso de Equipo de Protección Personal (EPP) en la agricultura Brasileña. La (in)adecuación de los equipos es evidente. Además de no proteger completamente al trabajador contra el pesticida, incluso agravar los riesgos, el EPP no elimina las condiciones insalubres como requiere la ley y también aumentan la probabilidad de contaminación de los trabajadores rurales en algunas actividades. Se discuten aspectos de la validación legal para la fabricación y venta de EPP. Este proceso de validación legal consiste en pruebas en laboratorios, por agentes agresivos aislados, que pueden ser considerados un hueco en el proceso de diseño. La validación legal no requiere el analisis de las actividades y/ o experimentos en situaciones de uso más frecuentes típicos o más críticos para la salud de los trabajadores.

Cet article analyse l'utilisation d'équipements de protection individuelle (EPI) dans l'agriculture du Brésil. L'insuffisance et l'(in)adaptation de l'équipement est évident. lls ne protègent pas entièrement le travailleur contre les pesticides et même aggravent les risques, les EPI ne supprime pas les conditions insalubres, comme l'exige la loi. Au contraire, ils augmentent la probabilité de contamination des travailleurs ruraux dans certaines activités. Ils ont discuté des aspects de la validation des EPI dans la législation brésilienne. Ce processus de validation pour la fabrication et la vente des EPI se compose de tests en laboratoire, par des agents agressifs isolés, qui peuvent être considérés comme une lacune dans le processus de conception. La validation ne 
demande pas l'analyse des activités et/ou des expériences dans des situations d'utilisation plus typiques et fréquentes ou les plus critiques pour la santé des travailleurs.

This article analyzes the use of Personal Protection Equipment (PPE) in the Brazilian Agriculture. The (in)adequacy of equipment is evident. They do not fully protect the worker against the pesticide and even aggravate the risks. PPE does not eliminate the unhealthy conditions as required by law and also increase the likelihood of contamination of rural workers in some activities. We discuss aspects of the legal validation of PPE. This process of legal validation for the manufacture and sale of PPE consists of tests in laboratories, by isolated aggressive agents, which may be considered a gap in the design process. The legal validation does not require the analysis of typical activities and/or experiments in more frequent use situations or most critical situations to the health of workers.

\section{ÍNDICE}

Mots-clés: équipement de protection individuelle (EPI), projet, agriculture Palavras-chave: equipamento de proteção individual (EPI), projeto, agricultura

Palabras claves: equipo de protección personal (EPP), proyecto, agricultura

Keywords: personal protective equipment (PPE), project, agriculture

\section{AUTORES}

\section{LUIZ ANTONIO MEIRELLES}

Escola Politécnica, Universidade Federal do Rio de Janeiro, Av. Horácio Macedo, 2030, sala G-209, Prédio do Centro de Tecnologia, Cidade Universitária, CEP 21941-914, Rio de Janeiro, Brasil. meirelles@ufrj.br

\section{MARCELO MOTTA VEIGA}

Escola Nacional de Saúde Pública, Fundação Oswaldo Cruz, Ministério da Saúde, Rua Leopoldo Bulhões, 1480/503 - Manguinhos, CEP 21.041-210, Rio de Janeiro, Brasil. mveiga@ensp.fiocruz.br \& Escola de Administração Pública, Universidade Federal do Rio de Janeiro, Rua Voluntários da Pátria, 107, Botafogo, Rio de Janeiro, CEP 22.270-000, marcelo.veiga@unirio.br

\section{FRANCISCO DUARTE}

Instituto Alberto Luiz Coimbra de Pós-Graduação e Pesquisa em Engenharia - (COPPE), Universidade Federal do Rio de Janeiro, Av. Horácio Macedo, 2030, G-209, Prédio do Centro de Tecnologia, Cidade Universitária, CEP 21941-914, Rio de Janeiro. duarte@pep.ufrj.br 